\title{
Metodología para el desarrollo de capacidades tecnológicas y de innovación para la puesta en marcha de una planta de tratamiento de agua residual doméstica municipal. Caso Tibasosa, Boyacá
}

\section{Methodology for the development of technological and innovation capabilities for a municipal domestic water treatment plant star-up. Case Tibasosa, Boyacá}

\author{
Ana María Hernández Abadía ${ }^{11}$ \\ Erika Sofía Olaya Escobar ${ }^{22}$ \\ Hugo Fernando Castro Silva ${ }^{33}$ \\ David Roberto Olaya Escobar ${ }^{44}$ \\ UPTC
}

1 ana.hernandez04@uptc.edu.co, Maestría en Administración, Universidad Pedagógica y Tecnológica de Colombia, Calle 4 Sur No. 15-134 Sogamoso (Boyacá), código postal:152211, Colombia. https://orcid.org/0000-0002-5923-1781.

$2 \quad$ erika.olaya@escuelaing.edu.co, Unidad de proyectos, Escuela Colombiana de Ingeniería Julio Garavito, AK.45 No.205-59 (Bogotá D.C), código postal: 110121, Colombia. https://orcid.org/0000-0001-6254-1169.

3 hugofernando.castro@uptc.edu.co, Escuela de ingeniería industrial, Universidad Pedagógica y Tecnológica de Colombia, Calle 4 Sur No. 15-134 Sogamoso (Boyacá), código postal:152211, Colombia. https://orcid.org/0000-0001-6020-402X $4 \quad$ d.olaya@javeriana.edu.co, Instituto de Salud Pública, Pontifica Universidad Javeriana. Cra 7 No 40-62. Edificio Pablo VI Piso 2. Bogotá Colombia código postal: 11001000, Colombia. https://orcid.org/0000-0002-5018-7454 . 


\section{RESUMEN}

Hoy prima la necesidad de garantizar la sostenibilidad y calidad del recurso hídrico, entendiendo que su gestión se deriva del ciclo hidrológico que vincula una cadena de interrelaciones entre diferentes componentes naturales y antrópicos, por lo que se asocia como un proceso de aprendizaje intertemporal del ser humano. La mayoría de los países le dan máxima prioridad al suministro de agua, dado que es una de las necesidades básicas humanas; sin embargo, según la UNESCO en el informe mundial sobre desarrollo de los recursos hídricos del 2019, un tercio de la población mundial no tiene acceso al agua potable administrada de forma segura. Este es el caso de varias comunidades del departamento de Boyacá, Colombia y en particular de las comunidades rurales del municipio de Tibasosa en donde, a pesar de contar con dos afluentes hídricos principales como el nacimiento "La mana del padre", y "El rio Chicamocha", que pueden suplir la necesidad hídrica; la fuente de abastecimiento se concentra en el rio Chicamocha, cuya disponibilidad y calidad del recurso hídrico es deficiente por ser uno de los ríos más contaminados de Colombia. El objeto de este estudio es la identificación de las capacidades que debe reunir una comunidad que tiene como propósito la puesta en marcha de una Planta de Tratamiento de Aguas Residuales (PTAR) domésticas, para lo cual se realizó una investigación en términos de características importantes tales como la formación técnica y humana, innovación y desarrollo tecnológico, organización social y del trabajo, planificación y gestión de calidad, sistematización-investigación e incidencia política y social. Posteriormente se llevó a cabo el planteamiento de una metodología para el manejo y desarrollo coordinado de las capacidades, con el propósito de gestionar el recurso hídrico en interacción con los demás recursos naturales, y propender por maximizar el bienestar social y económico resultante, en el área rural del municipio de Tibasosa, Boyacá.

Palabras clave: capacidades, innovación, tecnología, Planta de Tratamiento de Agua Residual, salud ambiental, gestión de recursos hídricos.

\section{ABSTRACT}

Globally, there is a need to guarantee the sustainability and quality of the water resource, understanding that its management derives from the hydrological cycle that links a chain of interrelationships between different natural and anthropic components, so it is associated as an intertemporal learning process of human beings. Most countries give the highest priority to water supply, given that it is one of the basic human needs, however, according to UNESCO in the 2019 world report on water resources development, a third of the world's population does not have access to safely managed drinking water. This is the case of several communities in the department of Boyacá, Colombia and in particular of the rural communities of Tibasosa municipality, where despite having two main water tributaries such as the source "La mana del padre", and "El rio Chicamocha ", which can meet the need for water; the supply source is concentrated in the Chicamocha river, whose availability and quality of water resources is deficient as it is one of the most polluted rivers in Colombia. The object of study is the identification of the capabilities that a community must have when its purpose is the start-up of a domestic Wastewater Treatment Plant (PTAR), for which an investigation was carried out in terms of important characteristics such as technical and human training, innovation and technological development, social and labor organization, quality planning and management, systematization - research and political and social impact. Subsequently, the approach of a methodology for the management and coordinated development of these capabilities was carried out, with the purpose of managing 
water in interaction with other natural resources, and striving to maximize the resulting social and economic wealth, in the rural area of Tibasosa, Boyacá.

Key words: capabilities, innovation, technology, Wastewater Treatment Plant, environmental health, water resource planning.

\section{INTRODUCCIÓN}

La disponibilidad de agua apta para el consumo humano a nivel mundial enfrenta un conjunto de problemas, los cuales en su conjunto se conocen como la problemática del agua (Pérez Lázaro, 2015), que es distinta en cada lugar del planeta.

Colombia no es ajena a esta situación, pues el manejo actual del agua en las zonas urbanas obedece principalmente a la prestación de los servicios públicos de acueducto y alcantarillado. Sin embargo, este manejo sectorial no responde a los desafíos que en materia de disponibilidad presente y futuro del recurso hídrico enfrenta el país, limitando el desarrollo socioeconómico, creando conflictos sociales y disminuyendo la sostenibilidad ambiental.

Si bien Colombia posee una gran riqueza hídrica, ésta no se encuentra distribuida de manera homogénea y contradictoriamente las zonas con mayor concentración de población son las más vulnerables. En parte, esta vulnerabilidad radica en que más del $80 \%$ de las cabeceras municipales se abastecen de fuentes de agua pequeñas, como arroyos, quebradas y riachuelos, que no garantizan la disponibilidad. Además, los grandes asentamientos humanos y los polos de desarrollo industrial, agrícola, pecuario e hidroenergético en el país, se han dado en regiones donde la oferta hídrica es menos favorable, lo que ha generado presiones sobre el recurso y señales preocupantes por los problemas de disponibilidad de agua en algunos municipios y áreas urbanas, en especial, durante periodos con condiciones climáticas extremas (Viceministerio de Ambiente, 2010).

Según el Instituto de Hidrología, Meteorología y Estudios Ambientales (IDEAM), la oferta hídrica en el país experimenta una disminución progresiva a causa de las limitaciones de uso por la calidad del agua, que es afectada por la contaminación que se deriva de las actividades socioeconómicas e industriales, por la carga de sedimentos generados en fenómenos erosivos y por los procesos propios de degradación de las cuencas (Zamudio Rodríguez, 2012). Respecto a la actividad contaminante de fuentes hídricas, se encontró que para el año 2016, la carga contaminante generada por el sector doméstico aumentó $16 \%$ respecto al año 2010 (564.109 toneladas/2010) y se removió sólo el $10 \%$ de esa carga, las regiones con mayor carga contaminante doméstica fueron: Bogotá, Antioquia, Valle del Cauca y Atlántico. En cuanto al sector industrial, se encontró que el $90 \%$ de la carga contaminante corresponde a materia orgánica, el $8 \%$ sólidos suspendidos y $2 \%$ nutrientes, las regiones con mayor carga contaminante industrial fueron: Bolívar, Valle del Cauca y Atlántico (IDEAM, 2018).

Según el Plan de Desarrollo Municipal de Tibasosa 2016 - 2019, actualmente en la zona urbana se cuenta con una cobertura del $95 \%$ de alcantarillado; sin embargo, estas aguas residuales no tienen ningún tipo de tratamiento y se vierten al rio Chicamocha, que es la misma fuente de abastecimiento, no solo para el mismo municipio de Tibasosa, sino también para el Municipio de Nobsa. Se debe hacer énfasis en el rezago que ha venido presentando por obras inconclusas en el cumplimiento a compromisos adquiridos con la autoridad ambiental, Corporación Autónoma Regional de Boyacá (Corpoboyacá), en el desarrollo de actividades en cuanto al Plan de Saneamiento y Manejo de Vertimientos (PSMV), cuyas causas son entre otras, las deficiencias en los programas para la 
protección del recurso hídrico en el municipio y la falta de compromiso institucional con el suministro óptimo del servicio de agua en el municipio. La primera fase de construcción de las Plantas de Tratamiento de Agua Residual (PTAR), se realizó mediante el contrato de obra No 021 de 2010, cuyo objeto fue: la construcción del alcantarillado sanitario y construcción de tres plantas de tratamiento de aguas residuales para mitigar el impacto ambiental generado sobre el Rio Chicamocha, pero aún no se ha terminado la ejecución de estas obras y por lo tanto no se ha cumplido con el objetivo propuesto (Alcaldía de Tibasosa, 2016). Adicionalmente en el plan de desarrollo $2020-2023$, se indica que hay insuficiente disponibilidad del recurso hídrico en el municipio y hace falta plantas de tratamiento de aguas residuales (PTAR) y plantas de tratamiento de agua potable (PTAP) en la mayoría de veredas (Alcaldía de Tibasosa, 2020).

De esta manera, la problemática se basa en que aun cuando hace parte del Plan de Saneamiento y Manejo de Vertimientos (PSMV) del municipio y se han venido adelantando labores para la construcción y puesta en marcha de una Planta de Tratamiento de Aguas Residuales (PTAR), actualmente no cuenta con una solución para sus vertimientos finales que contribuya a la descontaminación del río del que se abastece. Una planta de tratamiento de aguas residuales domésticas, le permitiría a la comunidad del municipio solucionar parcialmente el problema ambiental de recolección y tratamiento de las aguas residuales que actualmente se descargan al río Chicamocha, lo cual incide en la contaminación de los recursos naturales, la salud pública y al deterioro de la calidad de vida de los habitantes del municipio y usuarios del recurso aguas abajo. Además, de proveer a la comunidad de un sistema que pueda atender los patrones tecnológicos, sanitarios y ambientales requeridos y de propiciar al usuario un nivel de bienestar consistente con las características urbanas locales.

Según los criterios del Programa de las Naciones Unidas para el Desarrollo (PNUD), "el desarrollo de capacidades es el proceso mediante el cual las personas, organizaciones y sociedades obtienen, fortalecen y mantienen las aptitudes necesarias para establecer y alcanzar sus propios objetivos de desarrollo a lo largo del tiempo" (PNUD, 2009, p. 5). Es decir, la premisa consiste en buscar el desarrollo y fortalecimiento de las capacidades para la gestión del recurso hídrico, de tal forma que se favorezca en última instancia la gestión sustentable del agua y una mejora en el acceso al agua y al saneamiento por parte de todos los actores implicados. Es de suma importancia que no se desconozca la comunidad cuando se trate de buscar soluciones en la problemática de cuencas. Tanto los diagnósticos como las acciones correctivas deben ser concertadas con el fin de que la comunidad participe en la solución de sus propios problemas y los esfuerzos del estado sean reconocidos. Es necesario que la empresa privada, el gobierno y la comunidad se fusionen interviniendo en la consecución de planes y programas para una buena utilización del agua, realizando propuestas metodológicas que sean aplicadas en la gestión del recurso hídrico y particularmente en la puesta en marcha de la planta de tratamiento de aguas residuales para su municipio.

Domínguez \& Brown (2004), mencionan que las capacidades son habilidades necesarias para generar y administrar el cambio técnico, que incluyen destrezas, conocimientos y experiencias distintas de las requeridas para operar los sistemas técnicos. En ese sentido, se pretende que a través de la promoción del desarrollo de capacidades; la gestión del conocimiento, de innovación, gestión social y del recurso natural, se contribuya a la implementación de procesos para la mejora efectiva de las condiciones de 
vida de la comunidad de Tibasosa, que para este caso puntual se enfoca en el eficaz y eficiente tratamiento de los vertimientos finales al cuerpo receptor del cual también se abastece éste y otros municipios de la cuenca alta del río Chicamocha.

Desde este enfoque, el tipo de conocimiento que se busca producir y compartir es aquel que responde a las necesidades locales y promueve el fortalecimiento y desarrollo local, acotado en principio al municipio de Tibasosa, en el departamento de Boyacá (Colombia) pero perfectamente replicable a otras comunidades con características similares.

Este artículo pretende establecer una metodología para la identificación de capacidades para la puesta en marcha de una planta de tratamiento de aguas residuales en Tibasosa, Boyacá.

\section{METODOLOGÍA}

En esta sección se describe la metodología de revisión de literatura utilizada para identificar las diferentes orientaciones contextuales, alrededor del desarrollo de capacidades y de operación de plantas de tratamiento de agua residual. Así mismo se muestra la estrategia de abordaje de este tema.

Una revisión sistemática de literatura se define como "una manera de evaluar e interpretar toda la investigación disponible, que sea relevante respecto de una interrogante de investigación particular, en un área temática o fenómeno de interés" (Kitchenham, 2004). Esta metodología propone que la revisión se desarrolle en 3 etapas, planificación, desarrollo de la revisión, y publicación de resultados, donde en la etapa de planificación se debe especificar la necesidad de la revisión y la definición de un protocolo de búsqueda.

Por lo anterior en la etapa de planeación, se hizo necesario definir un protocolo de búsqueda, precisando los términos y sus combinaciones. Entre las palabras claves definidas se encuentra: desarrollo de capacidades, innovación, capacidades dinámicas, gestión estratégica, capacidades organizacionales, capacidades de innovación, modelos organizacionales, generación de conocimientos y recursos hídricos. De acuerdo con las palabras clave anteriores se construyó la ecuación de búsqueda, TS= ("capacity building" OR "capacity development") AND ("integrated management" OR "water resource"), se hizo uso de la base de datos Web Of Science y se obtuvieron 71 resultados (años 2002 - 2018). Para el desarrollo de la revisión se tuvo en cuenta que los artículos elegidos incluyeran información sobre el desarrollo de capacidades e innovación desde una perspectiva organizacional y/o institucional, siendo refinado por el año de publicación (2014 - 2018), tipo de documento (artículos) y área de investigación (gestión de recursos hídricos). Luego de la depuración de los resultados, se totalizaron las publicaciones útiles para el objeto de estudio, entre aquellos artículos seleccionados como resultado de la búsqueda y desarrollo de la revisión (7 resultados), y aquellos seleccionados por bola de nieve (2 documentos).

\section{RESULTADOS}

Los resultados se presentan en tres ítems, primero, la descripción y funcionamiento de una planta de tratamiento de agua residual; segundo, la identificación de características para el desarrollo de capacidades y tercero, la identificación de capacidades tecnológicas y de innovación para la puesta en marcha de una PTAR.

\subsection{Planta de tratamiento de agua residual}

El propósito de un proceso de tratamiento de agua residual es retirar el material contaminante, orgánico e inorgánico, el cual se puede encontrar en forma de sólidos 
suspendidos o disueltos. Lo anterior para lograr la obtención de una calidad de agua requerida por las normas de descarga o por la clase de reutilización que se le dará (Noyola et al., 2013) entre otros aspectos, la composición típica del agua residual cruda, la eficiencia de remoción de contaminantes por tipo de tecnología, indicadores de desempeño por tecnología, aspectos ambientales sobre localización y la estrategia espacial para la localización. Este artículo presenta la metodología desarrollada por los autores, construida con base en los aspectos técnicos, económicos y ambientales, como una herramienta para la toma de decisión de inversiones futuras de plantas de tratamiento de aguas residuales municipales con elementos multidisciplinarios.","author":[\{“dropping-particl e":"',"family":"Noyola","given":"Adalberto","nondropping-particle":"',"parse-names":false,"suffix":"”\},\{“dropping-particle":"',"family":"MorganS a g a s t u me", "given ": " Ju a n
Manuel","non-dropping-particle":",,"parsenames":false,"suffix":" $\},\{$ "droppingparticle":", "family":"Güereca","give n":"Leonor Patricia","non-droppingparticle":"","parse-names":false,"suffix":"'\}]," container-title":"Revista Tecnura","id":"ITEM1 ", " is sue ": " 46 ", " is sued": : "date parts": [ [ "2 20113 "] ] \}, "number-ofpages":"149","title":"Selección de tecnologías para el tratamiento de aguas residuales municipales: Guía de apoyo para ciudades pequeñas y medianas","type":"book","volu me":"19"\},"uris":["http://www.mendeley.com/ documents/?uuid=1 ba00a80-7c51-43ca-8e94b40277d1dd12"]\}],"mendeley":\{“formattedCit ation":"(Noyola et al., 2013. En la Figura 1 se representa el funcionamiento de una PTAR, donde se encuentra que, dependiendo de las condiciones iniciales del agua, esta deberá ser tratada con tecnologías distintas, y los parámetros estarán clasificados por la carga de materia orgánica inicial que traiga el agua.

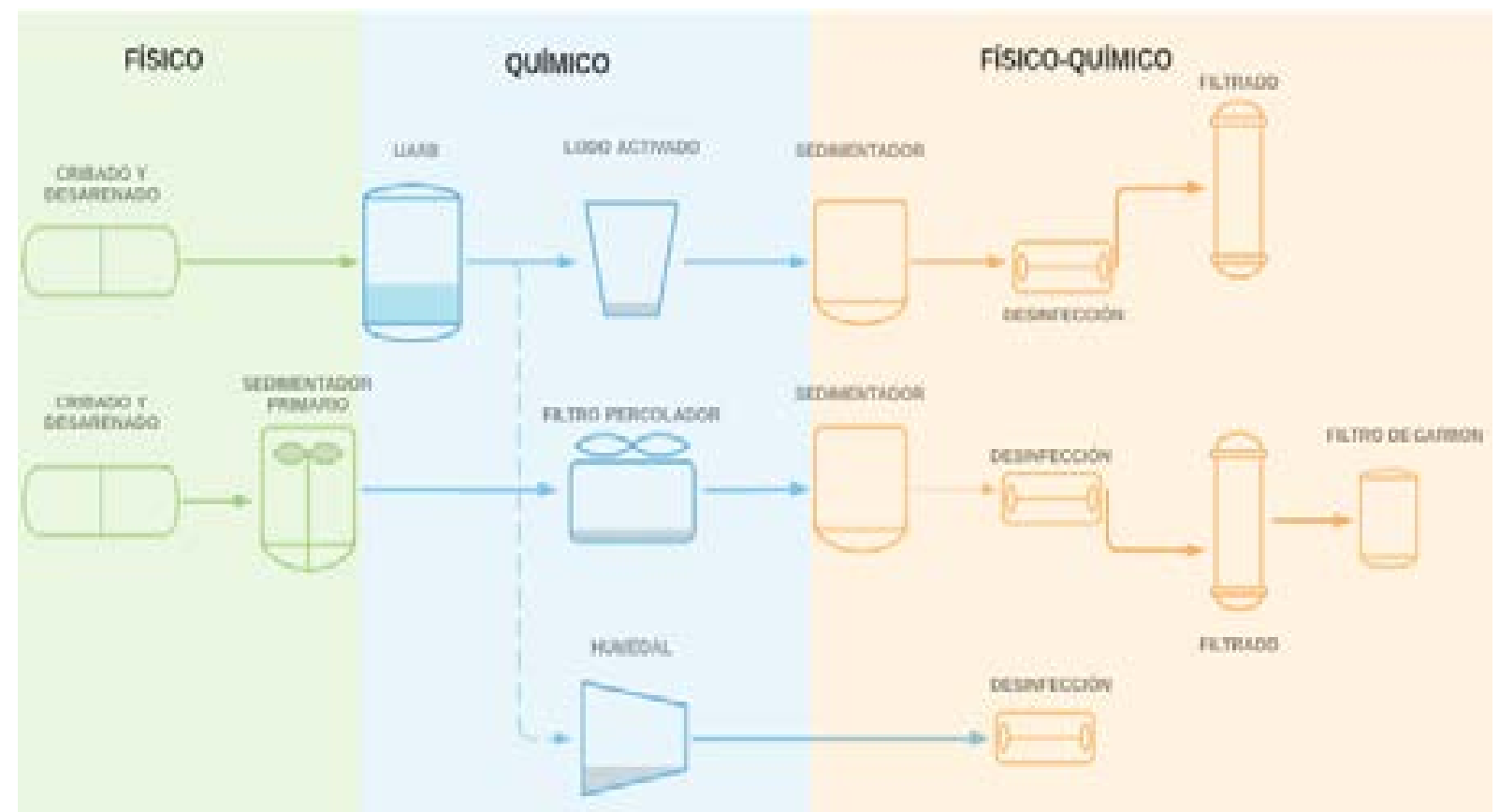

Figura 1. Esquema de Planta de Tratamiento de Agua Residual (PTAR)

Fuente: basado en Noyola et al., 2013entre otros aspectos, la composición típica del agua residual cruda, la eficiencia de remoción de contaminantes por tipo de tecnología, indicadores de desempeño por tecnología, aspectos ambientales sobre localización y la estrategia espacial para la localización. Este 
artículo presenta la metodología desarrollada por los autores, construida con base en los aspectos técnicos, económicos y ambientales, como una herramienta para la toma de decisión de inversiones futuras de plantas de tratamiento de aguas residuales municipales con elementos multidisciplinarios.","author":[[“dropping-particl e":"',",family":"Noyola","given":"Adalberto","nondropping-particle":"',",parse-names":false,"suffix":"'\},\{“dropping-particle":"',"family":"MorganS a g a s $\mathrm{t} u \mathrm{me}$ ", , g i ve n ": " Ju a n Manuel","non-dropping-particle":",",parsenames":false,"suffix":" $\},\{$ "droppingparticle":", "family":"Güereca","give n":"Leonor Patricia","non-droppingparticle":",,"parse-names":false,"suffix":"'\}]," container-title":"Revista Tecnura","id":"ITEM-
1 ", " is sue ": " 46", " is sued": : d a te -

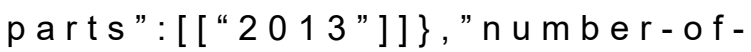
pages":"149","title":"Selección de tecnologías para el tratamiento de aguas residuales municipales: Guía de apoyo para ciudades pequeñas y medianas","type":"book","volu me":"19"\},"uris":["http://www.mendeley.com/ documents/?uuid=1 ba00a80-7c51-43ca-8e94b40277d1dd12"]\}],"mendeley":\{“formattedCitatio n":"(Noyola et al., 2013

En la Figura 2, se relacionan las tecnologías para el tratamiento de agua residual, identificando cinco sistemas de tratamiento de lodos agrupados en biológicos y fisicoquímicos, esta figura indica la amplia variedad de procesos unitarios para realizar el tratamiento de agua residual.

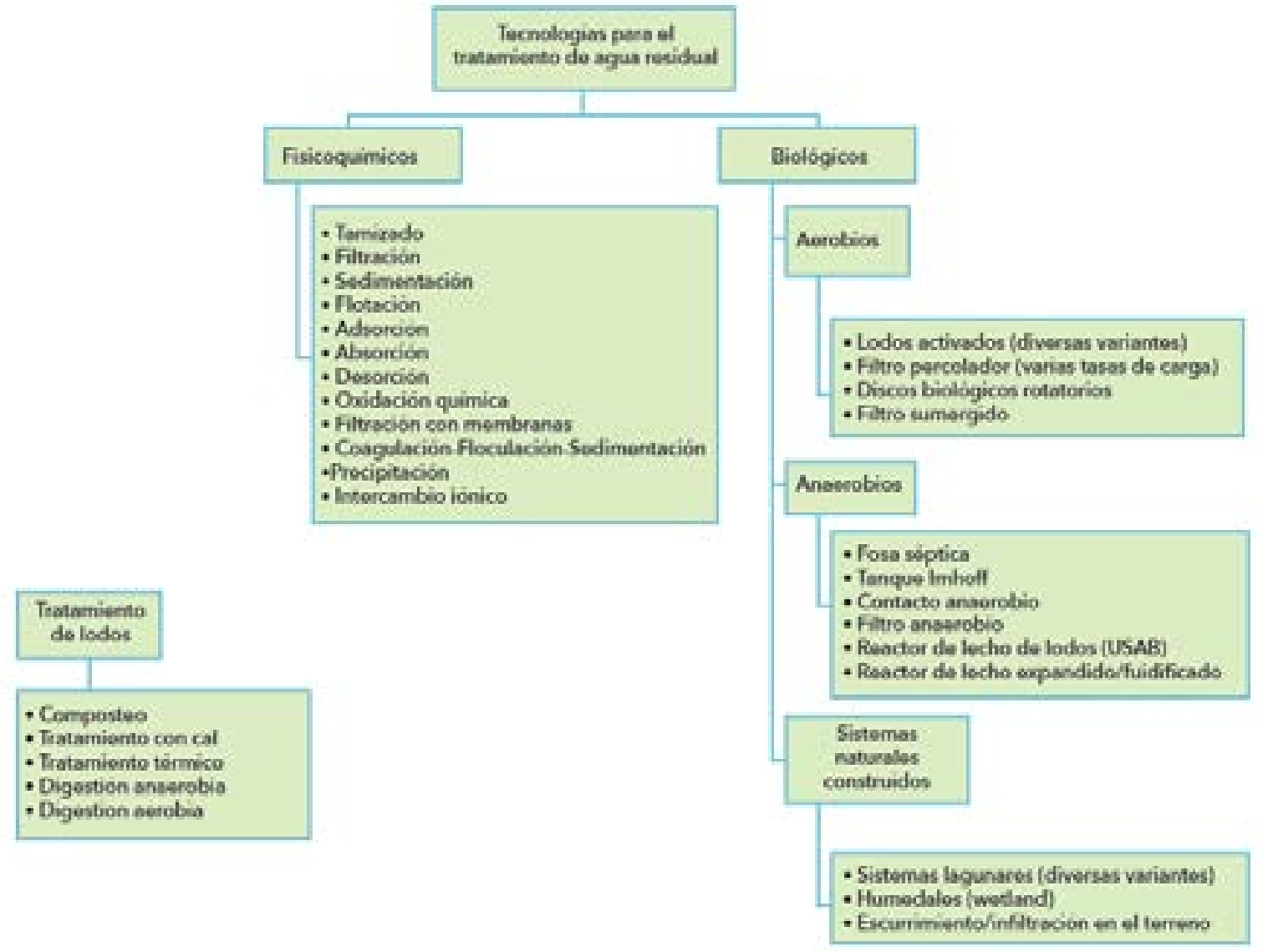

Figura 2. Clasificación de procesos para el tratamiento de aguas residuales

Fuente: Noyola et al., 2013 entre otros aspectos, la composición típica del agua residual cruda, la eficiencia de remoción de contaminantes por tipo de tecnología, indicadores de desempeño por tecnología, aspectos ambientales sobre localización y la estrategia espacial para la localización. Este 
artículo presenta la metodología desarrollada por los autores, construida con base en los aspectos técnicos, económicos y ambientales, como una herramienta para la toma de decisión de inversiones futuras de plantas de tratamiento de aguas residuales municipales con elementos multidisciplinarios.","author":[[“dropping-particl e":"',",family":"Noyola","given":"Adalberto","nondropping-particle":"',",parse-names":false,"suffix":"'”,\{“dropping-particle":"',",family":"MorganS a g a s t u me ", "g i ve n " : " J u a n Manuel","non-dropping-particle":",",parsenames":false,"suffix":" $\},\{$ "droppingparticle":", "family":"Güereca", "give n":"Leonor Patricia","non-droppingparticle":",,"parse-names":false,"suffix":"'\}]," container-title":"Revista Tecnura","id":"ITEM1 ", " is sue" : " 46 ", " is sued": \{ date -

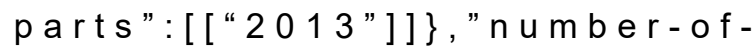
pages":"149","title":"Selección de tecnologías para el tratamiento de aguas residuales municipales: Guía de apoyo para ciudades pequeñas y medianas","type":"book","volu me":"19"\},"uris":[“http://www.mendeley.com/ documents/?uuid=1 ba00a80-7c51-43ca-8e94b40277d1dd12"]\}],"mendeley":["formattedCitatio n":"(Noyola et al., 2013

Por lo anterior, se encuentra que dependiendo del tipo de tecnologías de tratamiento de aguas residuales se requieren distintas capacidades, que permitan poner en marcha una PTAR, en este sentido se despliega a continuación la identificación de las mismas.

\section{2 . Identificación de características para el desarrollo de capacidades}

El planteamiento de metodologías para la puesta en marcha de una PTAR en Tibasosa, Boyacá, se basa en estrategias que agrupan características importantes para el desarrollo de las capacidades. De acuerdo con ALBOAN (2015), se encuentran: la formación técnica y humana, innovación y desarrollo tecnológico, organización social y del trabajo, planificación y gestión de calidad, sistematización-investigación e incidencia política y social.

- Formación técnica y humana: En el desarrollo de capacidades es la columna vertebral. Es necesario identificar en que procesos se va a intervenir y que se quiere transformar (ALBOAN, 2011). La idea de la formación es que el individuo adquiera habilidades y competencias que le permitan convertirse en un activo que facilite el logro de los objetivos, en este sentido, la educación es la principal herramienta para el desarrollo humano y la capacitación puede obtener cambios a nivel individual y social para mejorar la calidad de vida (Zahedi, 2012)economic, social, and cultural equality. [Ensieh zahedi.","author":[\{“dropping-pa rticle":",,"family":"Zahedi","given":"Ens ieh","non-dropping-particle":"”,"parsenames":false,"suffix":"”\}],"containertitle":"Life Science Journal","id":"ITEM1 ", " is sue" : " 4", " is sued": : "date parts":[["2012"]]\},"number-of-pages":"10978135","title":"Role of Professional and Technical Training in Human Devel opment","type":"report", "volume":"9 "],"uris":["http://www.mendeley.com/ documents/?uuid=a871d184-4a50-384fb139-e8d5f12b535e"]\}],"mendeley":\{“forma ttedCitation":"(Zahedi, 2012. La formación técnica y profesional es reconocida dentro de la planificación como un factor destacado para el desarrollo de regiones y países, porque aumenta la competitividad y contribuye a desarrollar la estructura productiva (UNESCO, 2019). Se pretende la adquisición de conocimientos y desarrollo de competencias, habilidades y actitudes. Se recomienda el uso de metodologías y marcos de trabajo ágiles como: Lean (Kaizen, Kaikaku, Shuhari, Monozukuri Genba y Poka Yoke), que busca eliminar las actividades que no aportan a la obtención de un producto o servicio; KanBan, que 
reduce la procrastinación en todas las áreas mediante el uso de tarjetas de gestión visual y Scrum, que busca entregar valor en cortos periodos de tiempo.

- Innovación y desarrollo tecnológico: La innovación incluye la idea de adquirir nuevas técnicas y conocimiento, pero también con la recuperación de saberes en lo local, esto significa poder anclar el conocimiento local con el desarrollo tecnológico con el fin de mejorar los sistemas de producción. Los recursos de conocimiento tecnológico tácito son los principales detonantes de la innovación y la innovación en este sentido no sólo depende del conocimiento interno, sino que está influida de forma positiva por los conocimientos externos (Díaz Díaz et al., 2006). Para la generación de innovación y desarrollo tecnológico es necesario realizar vigilancia tecnológica, y buscar transformar la información existente en conocimiento útil para los procesos (Vargas \& Castellanos, 2005).

- Organización social y del trabajo: la estrategia de organización social y del trabajo atiende a la dimensión organizativa de los colectivos a los que se acompaña, y se centra en el fortalecimiento de sus procesos organizativos. Tal fortalecimiento consiste en generar capacidades mediante formación (Zahedi, 2012) economic, social, and cultural equality. [Ensieh zahedi.","author":[\{"dropping-pa rticle":"","family":"Zahedi","given":"Ens ieh","non-dropping-particle":"", "parsenames":false, "suffix":""\}], "containertitle":"Life Science Journal","id":"ITEM1 ", " is sue " : " 4 ", " is sued " : \{ "d a te parts":["2012"]]\},"number-of-pages":"10978135","title":"Role of Professional and Technical Training in Human Devel opment", "type":"report", "volume" :"9 "\}, "uris":["http://www.mendeley.com/ documents/?uuid=a871d184-4a50-384fb139-e8d5f12b535e"]\}],"mendeley":\{"format tedCitation":"(Zahedi, 2012 que mejoren el desempeño interno de las organizaciones; por ejemplo, las capacidades para desarrollar un proyecto común, crear y consolidar espacios participativos de toma de decisiones, distribuir el conocimiento y el trabajo, solucionar conflictos, gestionar los recursos, etc. Esta estrategia pretende fortalecer a los colectivos como actores sociopolíticos y socioeconómicos, a fin de generar una fuerza político-económica que sea capaz de responder a sus necesidades básicas, personales, organizativas $\mathrm{y}$ comunitarias, considerando en ello la lucha por las transformaciones estructurales y contribuyendo a la construcción de una verdadera democracia. Esto está relacionado con la necesidad de generar capacidad para el cambio, la articulación y la concertación que permitan cambios sociales ALBOAN (2015).

- Planificación y gestión de calidad: Se refiere estrictamente a los procesos de gestión y calidad como parte fundamental en la función económico-productiva de la organización (ALBOAN, 2015). En este caso la organización hace referencia a la comunidad del municipio de Tibasosa Boyacá.

- Sistematización e investigación: La sistematización de procesos implica detenerse, reflexionar, extraer aprendizajes y tomar decisiones con base a lo aprendido que permitan mejorar la práctica. Es una metodología de investigación que se caracteriza por la construcción colectiva y participativa del aprendizaje, al incorporar a todas las personas y actores protagonistas en el proceso; y que enfatiza la lectura del contexto, motivando a tomar una mayor conciencia de la realidad para transformarla. 
Es indispensable para la transferencia de experiencias, ya sea en lo organizativo o en lo productivo, a otras personas y colectivos, así como para conseguir apoyos (ALBOAN, 2011).

- Incidencia política y social: Esta dirigida a influir sobre quienes toman las decisiones políticas de los poderes públicos, tanto a nivel local, regional, y nacional para presentar injusticias y realizar propuestas de políticas públicas, programas o proyectos que afecten la vida de las personas (ALBOAN, 2015).

\subsection{Identificación de capacidades de innovación y tecnológicas}

A partir de las variables relevantes para establecer la metodología, se han identificado las capacidades necesarias para la puesta en marcha de la PTAR. Dichas capacidades se presentan a continuación y se encuentran diferenciadas entre capacidades de innovación y capacidades tecnológicas.

\subsubsection{Capacidad de innovación}

La capacidad de innovación es importante para poner en marcha una PTAR, pues desde el punto de vista de la "capacidad", abarca tanto las habilidades "duras" (conocimiento específico técnico o especializado y el know-how, como la infraestructura, la tecnología o las finanzas) y las habilidades "blandas" o sociales (comunicación, liderazgo, procesos, etc.) que permiten a los individuos, las organizaciones, las redes y a los sistemas sociales más amplios, llevar a cabo sus funciones en la toma de decisiones, en la solución de problemas y alcanzar sus objetivos de desarrollo (Dourojeanni R., 2005). La innovación por su parte consiste en la introducción de un nuevo, o significativamente mejorado, producto (bien o servicio), de un proceso, de un nuevo método de comercialización o de un nuevo método organizativo, en las prácticas internas de la empresa, la organización del lugar, del trabajo o las relaciones exteriores (HELVETAS Swiss
Intercooperation, 2014), la innovación también puede ser en la gestión e incluye mejoras relacionadas con la manera de organizar los recursos para conseguir productos o procesos innovadores.

Teniendo en cuenta lo anterior, y de acuerdo con el Manual de Oslo (OCDE, 2018), las Capacidades de Innovación $(\mathrm{Cl})$ se podrían definir como un grupo de características propias de las organizaciones, entendidas como capacidades o habilidades mediante las cuales se puede lograr un mejor desempeño competitivo, a través de su correcta gestión. En el caso de este artículo, son las características propias de la comunidad del municipio de Tibasosa para poner en marcha la PTAR y desempeñarse de forma competitiva en el mejoramiento de la calidad del agua de su región.

Tradicionalmente los conceptos de innovación y $\mathrm{Cl}$ han sido abordados y analizados con un enfoque tecnológico, resaltando la importancia de los procesos de I+D y encontrando en éstos lo que representa en mayor parte las $\mathrm{Cl}$ y el éxito innovador. Adicional a estos procesos, se hace necesario tener en cuenta otras capacidades críticas en áreas como: producción, aprendizaje, asignación de recursos, mercadeo, organización, entre otras, las cuales toman protagonismo al influir también en el desempeño de las organizaciones.

Es posible encontrar en la literatura varias maneras de abordar el concepto de capacidades, que están estrechamente ligadas al desarrollo tecnológico y la innovación. Diversos autores las han definido en términos de las habilidades y conocimientos deunaorganizaciónqueposibilitan un mejor desempeño. Para Lall (1992)a simple framework for explaining the growth of national capabilities is set out, based on the interplay of incentives, capabilities and institutions. Each may suffer market failure and so require corrective intervention. The experience of some industrializing countries is described to assess 
the validity of this framework. It is concluded that interventions, carefully and selectively applied, are necessary for industrial success. (c) 1992.","author":[\{"dropping-particle":"'","fa mily":"Lall","given":"Sanjaya","non-droppingparticle":"',"parse-names":false,"suffix":""\}],"co ntainer-title":"World Development","id":"ITEM1 " , " is sue " : " 2 ", " is sued " : \{ "d a te parts":[["1992","2","1"]]\},"page":"165-186","pu blisher":"Pergamon", "title": Technologic al capabilities and industrialization","type":"art icle-journal","volume":"20"\},"uris":["http://www. mendeley. com/documents $/$ ?uuid $=5675159 \mathrm{~d}$ cda7-3554-9f19-1d7ba218de2c"]\}],"mend eley":\{"formattedCitation":"(Lall, 1992, las capacidades de innovación se definen como las habilidades y conocimientos necesarios para absorber efectivamente cierta información que permita mejorar las tecnologías existentes y crear nuevas tecnologías. El autor Kim (2000), citado por Gómez Rodríguez (2011), establece que la capacidad tecnológica se refiere al uso eficaz del conocimiento tecnológico con el propósito de mantener la competitividad en precio y en calidad. Esta capacidad admite que la organización asimile, emplee, adapte y modifique las tecnologías existentes, así como la creación de nuevas tecnologías y el desarrollo de nuevos productos y métodos de fabricación, todo esto para responder a los cambios del entorno.

Robledo Velásquez et al., (2008), hace un análisis de la literatura, proponiendo una clasificación de las capacidades de innovación basada en la propuesta de Yam et al., (2004) la cual se retoma y adapta al contexto del presente trabajo.

Tabla 1. Clasificación de capacidades de innovación

\begin{tabular}{|l|l|}
\hline Capacidad & Descripción \\
\hline Dirección Estratégica & $\begin{array}{l}\text { Capacidad para formular e implementar de manera adecuada } \\
\text { las estrategias que requiere la organización, incluyendo aquellas } \\
\text { estrategias o componentes de las mismas que sean necesarias para } \\
\text { construir una organización innovadora. }\end{array}$ \\
\hline I+D & $\begin{array}{l}\text { Capacidad para generar ideas; gestionar el portafolio de proyectos de } \\
\text { I+D+i; y proteger, valorar, negociar y contratar tecnología. }\end{array}$ \\
\hline Producción & $\begin{array}{l}\text { Capacidad para implementar las innovaciones en los procesos } \\
\text { productivos que satisfacen las necesidades del mercado. }\end{array}$ \\
\hline Aprendizaje Organización & $\begin{array}{l}\text { Capacidad para gestionar el conocimiento que proviene del ambiente } \\
\text { circundante y construir una organización que aprende. }\end{array}$ \\
\hline Gestión de Recursos & $\begin{array}{l}\text { Capacidad para identificar, adquirir y asignar apropiadamente los } \\
\text { recursos (capital, experiencia y tecnología a los procesos) necesarios } \\
\text { para innovar. }\end{array}$ \\
\hline Relacionamiento & $\begin{array}{l}\text { Habilidad para insertarse en los sistemas de innovación de diferente } \\
\text { orden (internacional, nacional, regional y sectorial), bajo los criterios } \\
\text { definidos por la estrategia empresarial y la Responsabilidad Social } \\
\text { Empresarial (RSE). }\end{array}$ \\
\hline
\end{tabular}

Fuente: Basado en Robledo Velásquez et al., (2008) y Yam et al., (2004) 
Se establecen entonces como preguntas metodológicas para el desarrollo de las capacidades de I+D:

\section{TIPO AMBIENTAL}

- ¿Puede operar la planta dentro de las fluctuaciones de temperatura del medio ambiente presentes en la región?

- ¿La dirección del viento dominante es favorable para la ubicación de la planta de tratamiento de aguas residuales en cuanto al transporte de aerosoles o posibles malos olores?

- ¿Se tiene una estimación de la generación de gases de efecto invernadero por parte del proceso de tratamiento (proveniente del agua y lodos)?

- ¿Se cuenta con un estudio de impacto ambiental, incluso preliminar, que valore los impactos de la operación de la planta de tratamiento de aguas residuales?

\section{Tipo técnico}

- Antes de montar la PTAR, ¿Se verificó que existen colectotes y redes de alcantarillados que conducirian las aguas servidas a la futura PTAR?

- ¿Se ha identificado, dentro de lo posible, alguna tecnología aplicable al caso y que haya sido desarrollada o adaptada en la región o en el país?

- ¿Se puede considerar que el agua residual por tratar es del tipo netamente municipal $\left(\mathrm{DBO}_{5}\right.$ total por debajo de los $\left.350 \mathrm{mg} / \mathrm{l}\right)$ ?

- ¿Se está seguro de que no hay descargas industriales de relevancia, o de cualquier otro tipo, que alteren el carácter municipal del agua residual?
- ¿La planta de tratamiento, preferentemente, integra o contempla el tratamiento mediante lodos activados por vía biológica?

- ¿En plantas pequeñas, se ha considerado dentro del tren de manejo de lodos, al menos para efectos de comparación y evaluación, los lechos de secado?

- ¿Se tiene contemplado como disponer adecuadamente los lodos generados?

- ¿La planta de tratamiento genera lodos susceptibles a ser usados como mejoradores de suelo o en la agricultura?

- ¿La planta de tratamiento integra o contempla el control de olores?

- ¿El control de olores hace uso de biotecnología o sistemas biológicos como biofiltros de composta?

- ¿La planta de tratamiento integra o contempla la mitigación de ruido?

- ¿Se tiene contemplado que hacer con el agua residual o agua parcialmente tratada durante el arranque de la planta o falla de la misma sin que afecte ostensiblemente al medio ambiente?

- ¿La planta de tratamiento usa equipamiento de fácil compostura y reposición?

- ¿Se considera que la tecnología usada en la planta de tratamiento favorece una fácil operación y mantenimiento de la misma?

- ¿Se considera que la planta no requiere personal altamente capacitado; ¿es decir, puede ser operada por un profesional de nivel técnico medio con la capacitación necesaria?

- ¿El consultor que propone la tecnología puede demostrar experiencia en su diseño y operación? 
- ¿En la tecnología propuesta se favorece el tratamiento biológico sobre los tratamientos físico-químicos?

- ¿Se ha considerado dentro del tren de tratamiento del agua, al menos para efectos de comparación y evaluación, un sistema anaerobio?

- ¿No hay dependencia de productos (enzimas, bacterias o microorganismos de cualquier tipo) que deban agregarse a la planta frecuentemente y generen dependencia económica?

- ¿Se reconoce que la planta de tratamiento genera lodos y su cantidad y calidad están determinadas y se ha considerado su manejo?

- Los diseños propuestos estab acordes con con elreglamento técnico para el sector de agua potable y saneamiento básico-RAS vigente.

\section{Tipo económico}

- ¿La tecnología utilizada en la planta de tratamiento puede contribuir de alguna forma con las actividades económicas de la región?

- ¿En cuanto a la reparación y/o mantenimiento de los equipos de la planta es posible apoyarse con prestadores de servicios de la región?

- ¿Se desglosa a detalle el costo de operación y mantenimiento de la planta de tratamiento?

- ¿El costo de operación y mantenimiento de la planta es sostenible considerando las finanzas del municipio o del organismo responsable de su operación?

\section{TIPO SOCIAL}

- ¿Ha sido consultada la población directamente relacionada o posiblemente afectada sobre la construcción de la planta de tratamiento?

- ¿Se ha hecho consciencia en la población sobre la necesidad y ventajas de contar con una planta de tratamiento de aguas residuales?

- ¿Se ha definido el esquema de participación ciudadana durante el proceso de toma de decisiones, así como para la adjudicación, construcción y operación?

- ¿Se considera contratar personal de la comunidad?

- ¿Se cuenta con un plan de apoyo para la educación de la comunidad (visitas guiadas, museo, servicio social, entre otras acciones)?

- ¿Se cuenta con un plan de capacitación para los empleados?

- ¿Se cuenta con un plan de respuesta a emergencias y brigadas de protección civil dentro de la planta?

\subsubsection{Capacidad tecnológica}

Para la puesta en marcha de la PTAR es de vital importancia la selección correcta de la tecnología que se va a utilizar, y cómo será el funcionamiento de ésta. A continuación, se presentan los títulos más importantes para el montaje adecuado de la PTAR y su funcionamiento (Noyola et al., 2013) entre otros aspectos, la composición típica del agua residual cruda, la eficiencia de remoción de contaminantes por tipo de tecnología, indicadores de desempeño por tecnología, aspectos ambientales sobre localización y la estrategia espacial para la localización. Este artículo presenta la metodología desarrollada por los autores, construida con base en los aspectos técnicos, económicos y ambientales, como una herramienta para la toma de decisión 
de inversiones futuras de plantas de tratamiento de aguas residuales municipales con elementos multidisciplinarios.","author":[[“dropping-particl e":"”,",family":"Noyola","given":"Adalberto","nondropping-particle":"',",parse-names":false,"suffix":"'”,\{“dropping-particle":"',"family":"MorganS a g a s t u me ", "g i ve n ": " J u a n Manuel","non-dropping-particle":",",parsenames":false,"suffix":" $\},\{$ "droppingparticle":", "family":"Güereca","give n":"Leonor Patricia","non-droppingparticle":",,"parse-names":false,"suffix":"'\}]," container-title":"Revista Tecnura","id":"ITEM1 ", " is sue" : " 46 ", " is sued": \{ "date parts": [ [ "2 01013 "] ] \}, "number-of pages":"149","title":"Selección de tecnologías para el tratamiento de aguas residuales municipales: Guía de apoyo para ciudades pequeñas y medianas","type":"book","volu me":"19"\},"uris":["http://www.mendeley.com/ documents/?uuid=1 ba00a80-7c51-43ca-8e94b40277d1dd12"]\}],"mendeley":;“formattedCitatio n":"(Noyola et al., 2013.

1. Proceso, aplicabilidad y funcionamiento: Esto estará dirigido estrictamente a cómo deberá adecuarse el proceso de acuerdo a las características particulares del agua residual por tratar y cuál será la calidad del agua tratada requerida (Noyola et al., 2013)entre otros aspectos, la composición típica del agua residual cruda, la eficiencia de remoción de contaminantes por tipo de tecnología, indicadores de desempeño por tecnología, aspectos ambientales sobre localización y la estrategia espacial para la localización. Este artículo presenta la metodología desarrollada por los autores, construida con base en los aspectos técnicos, económicos y ambientales, como una herramienta para la toma de decisión de inversiones futuras de plantas de tratamiento de aguas residuales municipales con elementos multidisciplinarios.","author":[\{“dr opping-particle":"',"family":"Noyola","given":"
Adalberto","non-dropping-particle":"',",parsenames":false,"suffix":" $\},\{$ "droppingparticle": ", "family": "MorganS a g a s tume ", "given ": " Ju a n Manuel","non-dropping-particle":"”,"parsenames":false,"suffix":" $\},\{$ "droppingparticle":", "family":"Güereca","give n":"Leonor Patricia","non-droppingparticle":"',",parse-names":false,"suffix":"'”],", container-title":"Revista Tecnura","id":"ITEM1 ", "is sue": " 46", "is sued": : "dateparts": [ [ "2013"] ] 013 , "number-ofpages":"149","title":"Seleccióndetecnologías para el tratamiento de aguas residuales municipales: Guía de apoyo para ciudades pequeñas y medianas","type":"book","volu me":"19"\},"uris":[“http://www.mendeley.com/ documents/?uuid=1 ba00a80-7c51-43ca8e94-b40277d1dd12"]\}],"mendeley":\{“form attedCitation":"(Noyola et al., 2013. Para lo anterior se ven involucrados otros factores que se presentan a continuación:

\section{- Intervalos de flujos en los que el} sistema funcionará: Esta es una de las variables más importantes ya que el sistema funciona adecuadamente en intervalos de caudal estrictos.

\section{- Tolerancia a las variaciones de}

flujo: Es necesario que el sistema tenga una tolerancia a las variaciones de flujo del sistema, en épocas de sequía y épocas de lluvia los flujos tendrán variaciones que deben ser aceptadas adecuadamente por el sistema y no interferir con el funcionamiento adecuado de la planta.

\section{- Características del agua residual:} las características del efluente es una de las variables importantes en la selección del proceso y los requerimientos en la operación. La disponibilidad de nutrientes para los procesos biológicos es un factor crítico, si contiene mucha carga orgánica y podría inhibir el proceso. 
- Eficacia de la remoción: Está directamente ligada a la legislación vigente en donde se va a llevar a cabo la operación. Los tipos y cantidades de residuos sólidos, líquidos o gaseosos generados por un proceso de tratamiento deben ser conocidos o estimados. Además, debe considerarse la disposición final de todos los residuos que puedan salir de la operación.

2. Generación de residuos: El uso que se le dará a los residuos después de su uso. La utilización de gases como energía renovable, los lodos como fertilizantes o estabilizantes de suelos.

3. Aceptación por parte de la comunidad: Este es un factor decisivo para que se realice o no la construcción de la PTAR. Una obra debe ser aceptada por la organización a la que se le dará el servicio (población, industria, etc.) y vecinos.

4. Generación de subproductos con valor económico o de uso: En algunos casos las PTAR pueden generar como subproductos, residuos que tendrán algún valor económico que contribuya directamente a la sustentabilidad. Entre ellos podemos tener lodos para utilizarlo como inoculo en otras plantas, o como para utilizarlo como fertilizantes de suelos, en el mejor de los casos biogás para utilizarlo como valor energético.

5. Requerimientos de área: Es necesario, tener claro el área necesaria para la construcción de la planta ya que este puede ser un factor fundamental, debido a la poca disponibilidad del terreno, o los altos costos de este puede influir en la factibilidad del proceso. El estudio del terreno, y el suelo es fundamental para este tipo de proyectos. En este aspecto pueden funcinar muy bien las asociaciones público-privadas, donde el estado puede ser el dueño del terreno y un consescionario puede encargarse de la infrestructura, equipos y tecnología.

6. Costos: El estudio de costos del proyecto en el análisis financiero será definitivamente una de las variables más importantes en el momento de escoger la tecnología y esta determinará el costo real del valor tratado de agua por metro cúbico. En estos costos también estarán incluidos el tema de la inversión y el tema del mantenimiento en el tiempo de la vida útil de la planta.

Inversión inicial (infraestructura y puesta en marcha de la planta).

\section{Costos de mantenimiento y} operación: Costos de insumos (reactivos), Costos de energía, gastos administrativos y de personal.

7. Diseño y construcción: Para este aspecto es necesario el diseño, la experiencia del contratista y la tecnología seleccionada, la complejidad en la construcción y el equipamiento.

8. Operación: El funcionamiento de la planta que garantiza la producción de agua tratada deseada bajo la legislación regida. Para garantizar esto es necesario tener en cuenta la flexibilidad de la operación (las variaciones que acepta el sistema en operación y la capacidad de actualización y ampliación de la planta); confiablidad del proceso (la calidad del agua y la cantidad sea repetitiva y siempre cumpla los parámetros de la legislación); complejidad de la operación (Es necesario establecer la complejidad de la operación en condiciones normales y en condiciones adversas para establecer el perfil y número de personal requerido en la planta. Se deben establecer puntos de control y control de calidad con laboratorios analíticos); requerimientos de 
personal (según operación, tamaño de la planta y perfiles).

\section{Entorno e impacto al medio ambiente:} Las variables ambientales que se deben controlar en la operación de la planta y la afectación del medio ambiente circundante. Se tiene entonces la temperatura (Las variaciones pueden afectar el metabolismo de los microorganismos y la capacidad de operación de la planta); producción de ruido (los equipos que produzcan ruido pueden causar mala aceptación por parte de la comunidad, adicional a los requerimientos de salud y seguridad industrial en el personal); contaminación visual ( Se evalúa el diseño arquitectónico de la planta y su integración con la arquitectura de la región y del paisaje del sitio); producción de malos olores (sobre todo cuando hay asentamientos humanos cercanos, puede causar problemas en su aceptación. Es necesario el uso de membranas que controlen olores); generación de gases de efecto invernadero (su disminución puede estar ligada a el uso de tratamientos biológicos y el aprovechamiento de energías renovables con residuos producidos dentro de la planta); condiciones para la reproducción de insectos y animales dañinos, que pueden ser vectores para enfermedades y problemas de salud pùblica. Emisiones de bioaerosoles que pueden ser altamente perjudiciales para las comunidades vecinas a la planta.

Noyola et al., (2013)entre otros aspectos, la composición típica del agua residual cruda, la eficiencia de remoción de contaminantes por tipo de tecnología, indicadores de desempeño por tecnología, aspectos ambientales sobre localización y la estrategia espacial para la localización. Este artículo presenta la metodología desarrollada por los autores, construida con base en los aspectos técnicos, económicos y ambientales, como una herramienta para la toma de decisión de inversiones futuras de plantas de tratamiento de aguas residuales municipales con elementos multidisciplinarios.","author":[\{“dropping-particl e":"',"family":"Noyola","given":"Adalberto","nondropping-particle":"',"parse-names":false,"suffix":"'”,\{“dropping-particle":"',"'family":"MorganS a g a s t u m e ", "g i ve n " : " Ju a n Manuel","non-dropping-particle":"”,"parsenames":false,"suffix":" $\},\{$ "droppingparticle":", "family":"Güereca","give n":"Leonor Patricia","non-droppingparticle":"”,"parse-names":false,"suffix":"”\}]," container-title":"Revista Tecnura","id":"ITEM1 ", " is sue ": " 46 ", "is sued": \{ "date parts": [ [ "2 2013 " ] ] \}, "number-of pages":"149","title":"Selección de tecnologías para el tratamiento de aguas residuales municipales: Guía de apoyo para ciudades pequeñas y medianas","type":"book","volu me":"19"\},"uris":["http://www.mendeley.com/ documents/?uuid=1 ba00a80-7c51-43ca-8e94b40277d1dd12"]\}],"mendeley":\{“formattedCitatio n":"(Noyola et al., 2013 establece un diagrama de flujo que puede ser utilizando en este caso para la selección de tecnologías para el tratamiento del agua en la PTAR de Tibasosa, Boyacá. 


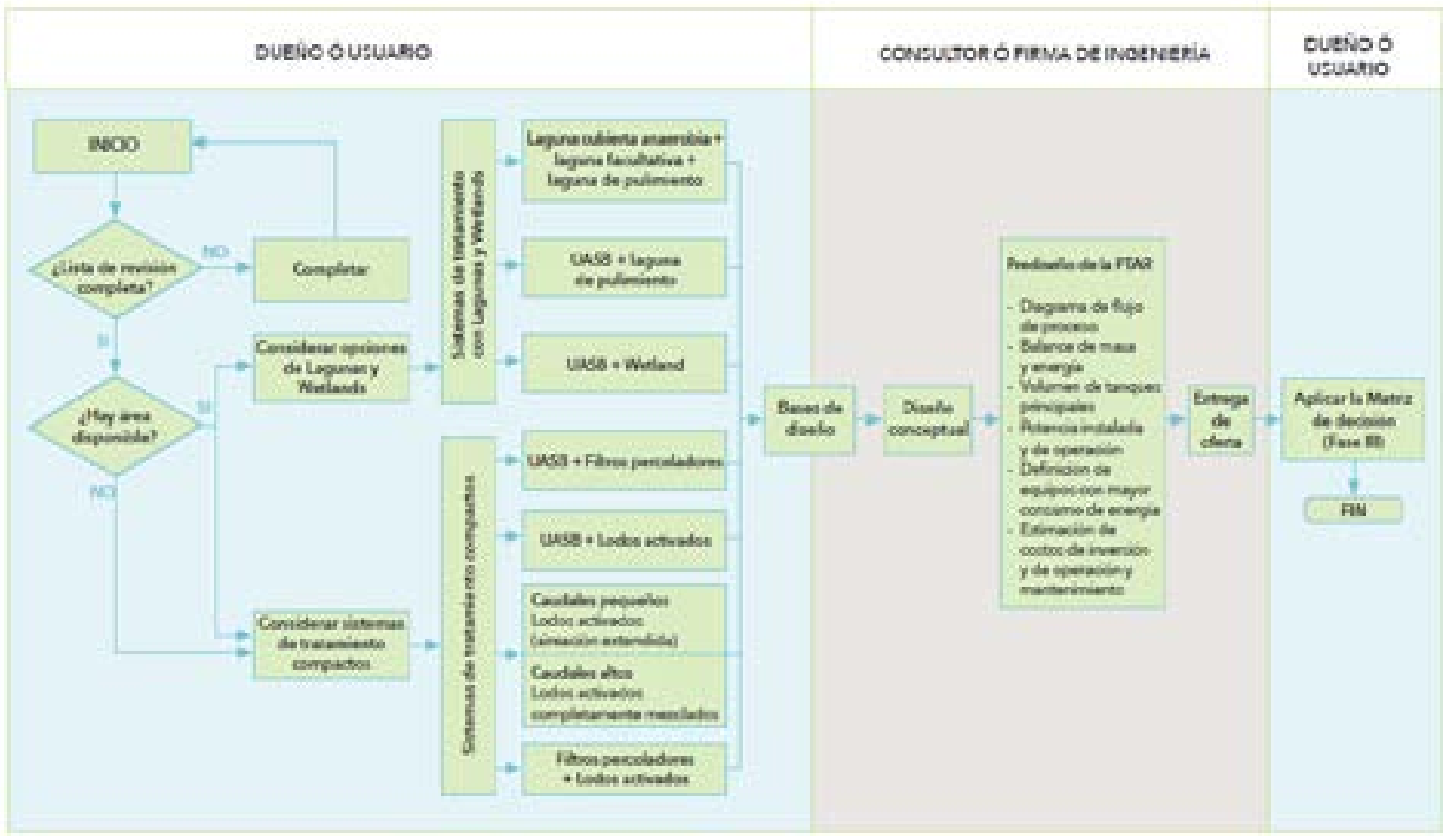

Figura 3. Esquema para la toma de decisiones en la selección de la tecnología adecuada

Fuente: Noyola et al., 2013entre otros aspectos, la composición típica del agua residual cruda, la eficiencia de remoción de contaminantes por tipo de tecnología, indicadores de desempeño por tecnología, aspectos ambientales sobre localización y la estrategia espacial para la localización.

Esteartículopresentalametodologíadesarrollada por los autores, construida con base en los aspectos técnicos, económicos y ambientales, como una herramienta para la toma de decisión de inversiones futuras de plantas de tratamiento de aguas residuales municipales con elementos multidisciplinarios.","author":[\{“dropping-particl e":"”,"’family":"Noyola”,"given":"Adalberto","nondropping-particle":"',"parse-names":false,"suffix":"'”,\{“dropping-particle":"',"family":"MorganS a g a s $t$ u me ", "give n": " J u a n Manuel","non-dropping-particle":",",parsenames":false,"suffix":" $\},\{$ "droppingparticle":", "family":"Güereca","give n":"Leonor Patricia","non-droppingparticle":"',"parse-names":false,"suffix":"”\}]," container-title":"Revista Tecnura","id":"ITEM1 ", , is sue " : " 46 ", " is sued": \{ d a te -

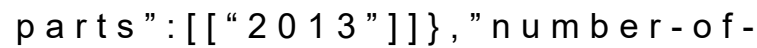
pages":"149","title":"Selección de tecnologías para el tratamiento de aguas residuales municipales: Guía de apoyo para ciudades pequeñas y medianas","type":"book","volu me":"19"\},"uris":["http://www.mendeley.com/ documents/?uuid=1 ba00a80-7c51-43ca-8e94b40277d1dd12"]\}],"mendeley":\{“formattedCitatio n":"(Noyola et al., 2013

\section{CONCLUSIÓN}

Se busca impulsar la adecuada gestión y tratamiento de aguas residuales en la región, implementando sistemas que reduzcan el impacto ambiental y social que se presentan en el municipio de Tibasosa y sus alrededores, promoviendo la capacitación de operarios para el óptimo funcionamiento de estas plantas de tratamiento. La idea de este artículo es ayudar a establecer la metodología para la identificación de las capacidades y la adecuada gestión de los servicios de una planta de tratamiento de agua residual para el municipio de Tibasosa, 
Boyacá. Para ello se consideró plantear las tecnologías existentes, y hacer énfasis en que es necesario el estudio inicial del agua para la buena selección de tecnologías. Como empresa y organización es necesario entender que todo el personal debe estar preparado y actualizado en las tecnologías que esta llevará a cabo, así mismo la selección del personal será fundamental para el buen funcionamiento de la PTAR. La metodología está basada en entender el entorno, la tecnología y la comunidad, sus requerimientos, necesidades y cómo éstas pueden afectar el territorio circundante.

Aunque la naturaleza aplicada de esta investigación se centró en el caso del Municipio de Tibasosa, es importante resaltar que los principios y fundamentos derivados de este trabajo tienen aplicación de interes general. Lo cual permite aplicar los hallazgos a otros contextos tanto regionales como internacionales.

\section{REFERENCIAS BIBLIOGRÁFICAS}

ALBOAN. (2011). El desarrollo alternativo por el que trabajamos. Aprendizajes desde la experiencia. $\mathrm{http}: / /$ centroderecursos. alboan.org/es/registros/6239-el-desarrolloalternativo-por

ALBOAN. (2015). El desarrollo de capacidades como estrategia de cambio Una experiencia colectiva de búsqueda de alternativas. Investigaciones ALBOAN, 1(1), 25-32. www.alboan.org

Alcaldía de Tibasosa. (2016). Plan de desarrollo municipal 2016 - 2019. https:// tibasosaboyaca.micolombiadigital. gov.co/sites/tibasosaboyaca/content/ files/000021/1033_1-plan-de-desarrollotibasosa-20162019.pdf

Alcaldía de Tibasosa. (2020). Plan de desarrollo territorial 2020-2023. www.tibasosaboyaca.gov.co.
Díaz Díaz, N. L., Aguiar Díaz, I., \& De Saá Pérez, P. (2006). El conocimiento organizativo tecnológico y la capacidad de innovación. Evidencia para la empresa industrial española. Cuadernos de Economía y Dirección de La Empresa.

Domínguez, L., \& Brown, F. (2004). Medición de las capacidades tecnológicas en la industria mexicana. Revista de La CEPAL.

Dourojeanni R., A. (2005). Desafíos para la gestión integrada de los recursos hídricos. Intern Ational W a Ter Resources Association (IWRA).

Gómez Rodríguez, M. E. (2011). Evolución de las capacidades de innovación en la industria colombiana: Un análisis comparativo de los resultados de las encuestas de innovación de 1996 y 2005 [Universidad Nacional de Colombia]. https://repositorio. unal.edu.co/bitstream/handle/unal/8615/ Gomez\%26Robledo.2011.Capacidades de_innovación_en_la_industria_ colombiana.pdf?sequence $=1$ \&isAllowed $=y$

HELVETAS Swiss Intercooperation. (2014). Desarrollo de capacidades y competencias para la gestión territorial concertada de los recursos naturales. Aplicación del modelo mariposa.

IDEAM. (2018). Reporte de avance del estudio nacional del agua 2018. In Cartilla ENA 2018.

Kim, L. (2000). La dinámica del aprendizaje tecnológico en la industrialización. Seúl, Edit. Universidad de Korea. http://www.oei. es/historico/salactsi/limsu.pdf 
Kitchenham,B.(2004). Procedures for Performing Systematic

Reviews.http://csnotes.upm.edu.my/kelasmaya/pgkm20910

nsf/0/715071a8011d4c2f482577a700386d3a/\$FILE/10.1.1.122.3308\%5B1\%5D.pdf

Lall, S. (1992). Technological capabilities and industrialization. World Development, 20(2), 165-186. https://doi.org/10.1016/0305-750X(92)90097-F

Noyola, A., Morgan-Sagastume, J. M., \& Güereca, L. P. (2013). Selección de tecnologías para el tratamiento de aguas residuales municipales: Guía de apoyo para ciudades pequeñas y medianas. In Revista Tecnura (Vol. 19, Issue 46). https://doi.org/10.14483/udistrital.jour.tecnura.2015.4.a12

OCDE. (2018). Manual de Oslo 2018 (The Measurement of Scientific, Technological and Innovation Activities). OCDE. https://doi.org/10.1787/9789264304604-en

Pérez Lázaro, R. (2015). La nueva cultura del agua, el camino hacia una gestión sostenible. Causas e impactos de la crisis global del agua. Cuadrenos de Trabajo NO. 68. Insittuto de Estudios Sobre Desarrollo y Cooperación Internacional, 53.

PNUD. (2009). Desarrollo de capacidades: texto básico del PNUD. Grupo de Desarrollo de La Capacidad. http://www.unpcdc.org/media/220984/capacity_development_a_undp_primer_spanish.pdf

Robledo Velásquez, J., Gómez Jiménez, F. A., \& Restrepo Arias, J. F. (2008). Relación entre capacidades de innovación tecnológica y desempeño empresarial e Colombia. Memorias Del Primer Congreso Internacional de Gestión Tecnológica e Innovación. Universidad Nacional de Colombia, August 2008.

UNESCO. (2019). EDUCACIÓN Y FORMACIÓN TÉCNICA Y PROFESIONAL. https://www.siteal. iiep.unesco.org/sites/default/files/sit_informe_pdfs/siteal_educacion_y_formacion_tecnica_ profesional_20190607.pdf

Vargas, F., \& Castellanos, O. (2005). Vigilancia como herramienta de innovación y desarrollo tecnológico. Caso de aplicación: Sector de empaques plásticos flexibles. Ingeniería e Investigación, 25. http:// www.redalyc.org/articulo.oa?id=64325205

Viceministerio de Ambiente. (2010). Política Nacional para la Gestión Integral del Recurso Hídrico.

Yam, R. C. M., Guan, J. C., Pun, K. F., \& Tang, E. P. Y. (2004). An audit of technological innovation capabilities in Chinese firms: Some empirical findings in Beijing, China. Research Policy, 33(8), 1123-1140. https://doi.org/10.1016/j.respol.2004.05.004

Zahedi, E. (2012). Role of Professional and Technical Training in Human Development. In Life Science Journal (Vol. 9, Issue 4). http://www.lifesciencesite.comhttp//www.lifesciencesite.com.57

Zamudio Rodríguez, C. (2012). Gobernabilidad sobre el recurso hídrico en Colombia: entre avances y retos. Gestión y Ambiente, 15(3), 99-112. https://revistas.unal.edu.co/index.php/gestion/article/ view/36284 